\title{
Lived Experience in Patients with Recurrent Glioblastoma in Japan: A Narrative Study
}

Asian/Pacific Island Nursing Journal

Volume 2(4): 157-165

(C)Author(s) 2017

http://digitalscholarship.unlv.edu/apin/

\section{Ai Chikada ${ }^{a}$, Asako Takekuma Katsumata ${ }^{b}$, Mariko Asase ${ }^{a}$, Sayaka Takenouchic, Yoshiki Arakawa $^{d}$, and Kazuko Nin ${ }^{a}$}

\begin{abstract}
Glioblastoma (GBM) is well known to have one of the poorest prognoses among all cancers. Patients with GBM in progression-free survival (PFS) may be relatively stable and can often maintain their quality of life. Thus, PFS is a desirable goal. In Japan, the median PFS is 11 months. It is difficult to grasp a patient's thoughts and hopes when, after PFS, they are readmitted due to recurrence or acute deterioration. Therefore, this study aimed to describe the lived experience of illness in patients with recurrent GBM, focusing on PFS. We enrolled five patients into the study; however, only four patients completed data collection. Data were collected using semi-structured interviews. We also conducted a thematic narrative analysis. As a result, we generated one overall theme: Even in vulnerable and constrained daily lives, the aim was gaining a sense of stability — and maintaining it steadily — as far as possible, on their own. That sense of stability is fragile so that maintaining equilibrium is a precarious enterprise. Moreover, in PFS, participants were trying to maintain equilibrium by reevaluating themselves and sometimes giving up something, although they received support from people around them. We infer that it is important for nurses to assess and understand the fluctuations in that sense of stability through continuous involvement with patients. An interdisciplinary approach and lateral integration of care are important to meet the needs of GBM patients. This understanding will lead to nursing supports that help patients live with stability, pride, and dignity.
\end{abstract}

Keywords: glioblastoma, high grade glioma, malignant brain tumor, narrative, qualitative study

Gliomas account for $28.3 \%$ of primary brain tumors. In Japan, gliomas occur at a rate of 6.6 per 100,000 persons (Committee of Brain Tumor Registry of Japan, 2014). According to the World Health Organization, gliomas Grades III and IV are malignant. Glioblastoma (GBM; Grade IV) is known to have one of the poorest prognoses among all cancers, and the median survival rate is $12-15$ months (compared with 2-5 years for anaplastic astrocytoma, Grade III; Pompili, Telera, Villani, \& Pace, 2014). Reports show that of the 1,381 newly diagnosed GBM patients in Japan, 52.9\% already have a severe neurological deficit (Narita \& Shibui, 2015); the onset of disease may be acute or insidious (Cahill \& Armstrong, 2011). Treatment extends the overall survival rate. However, worsening of existing neurological deficits, the appearance of new symptoms and treatment-associated symptoms may compromise their quality of life (Cahill \& Armstrong, 2011).
Although tumor control or shrinkage by initial treatment may improve signs, symptoms, and activities of daily living (ADL; Cahill \& Armstrong, 2011; Higuchi, Tanaka, Noguchi, \& Kusumi, 2006; Tsumura, Yamazaki, \& Kamibeppu, 2010), tumor recurrence is frequently unavoidable and incurable (Tsumura et al., 2010).

\footnotetext{
${ }^{a}$ Department of Human Health Sciences, Graduate School of Medicine, Kyoto University, Japan

${ }^{b}$ Division of Health Innovation and Nursing, Faculty of Medicine, University of Tsukuba, Japan

${ }^{c}$ Department of Ethics Support, Kyoto University Hospital, Japan

${ }^{d}$ Department of Neurosurgery, Graduate School of Medicine,

Kyoto University, Japan

Corresponding Author:

Kazuko Nin, RN., Ph.D.

Department of Human Health Sciences, Graduate School of Medicine, Kyoto University, Japan

Email: nin@kuhp.kyoto-u.ac.jp
} 
Maintaining a relatively stable quality of life without progression, regardless of how long it may last, is important for the patients considering GBM's prognosis including the likelihood of additional neurological deficits once recurrence starts. The median progression-free survival (PFS) period is 11 months (Committee of Brain Tumor Registry of Japan, 2017). When these patients are readmitted due to tumor recurrence or if the tumor deteriorates because of neurological deficits, it is crucial to grasp the nature of their thoughts and hopes because these thoughts and hopes may guide nurses to provide better care in their remaining time.

Kleinman (1988) stated that illness is a lived experience; it can be narrated, and when caregivers understand its narrative patterns, they can optimally engage with the patient's experience. Furthermore, Kleinman (1988) adds, "Legitimating the patient's illness experience-authorizing that experience, auditing it empathically - is a key task in the care of the chronically ill" (p. 17). That legitimizing of experience requires enlightened listening to the narratives of patients with GBM throughout the trajectory of their illness. Therefore, nurses can intervene so that patients have quality time in PFS.

Patients with high-grade glioma (HGG) are reported to experience loss of self, a sense of waiting and uncertainty, and focus on immediate needs in their illness trajectory (Philip et al., 2014). Other research has reported that HGG patients with neurological dysfunction suffer from the threat of a malignant brain tumor, confrontation with death, loss of feelings of self, loss of dignity, and a decline in self-reliance (Kamma, Sato, \& Kuwabara, 2008). Sterckx et al. (2013) conducted a systematic review, which analyzed 16 qualitative studies of the impact of $\mathrm{HGG}$ on patients and caregivers' everyday lives. Results found that at diagnosis, HGG patients felt shocked and recognized death. During different phases of the disease, patients felt uncertain about the effect HGG would have on their quality of life and future, and they suffered from the loss of autonomy. In such circumstances, they needed hope, support, and information. However, these studies included HGG patients in various phases of the disease trajectory. For example, Umeda and Iwata (2015) reported that middle-aged HGG patients in PFS - including three GBM patients-were seeking a way of life that would accommodate this critical, life-threatening experience, and the crisis of identity it brought. Furthermore, Iwata (1998) reported on a neurosurgeon and associate professor at a university hospital who suffered from GBM in his 40s, who recorded what he learned and felt after he became a patient. He felt fear that he could expect in detail what would happen to him in his illness trajectory and realized that there was a gap between patient's need and actual medical care. As
Kleinman (1988) observed, "The trajectory of chronic illness assimilates to a life course, contributing so intimately to the development of a particular life that illness becomes inseparable from life history" (p. 8). Thus, to understand the subjective experiences of patients with GBM, it is necessary to understand them in their life context. We found no studies that described the subjective experience of GBM patients in PFS and considered the individual voices of patients. Consequently, this study aimed to describe the lived experience of illness in patients with recurrent GBM, focusing on the PFS stage of their lives.

\section{Method}

In this study, we defined the lived experience of illness as "How patients perceive, live with, and respond to symptoms and disability" (Kleinman, 1988, p.3). We obtained ethical approval from the Kyoto University Graduate School and Faculty of Medicine, Kyoto University Hospital, Ethics Committee (R04730-1).

\section{Design}

We used a qualitative narrative study design to conduct the interviews: "Narrative inquiry enables participants to tell the story of their experience and provides an approach to the way people create meaning in their lives by the way they construct their narrative" (Davies, Crowe, \& Whitehead, 2016, p, 534). Narrative inquiry elucidates the participant's subjective experience.

\section{Participants}

Participants who were diagnosed with GBM recurrence and notified of their disease and its prognosis were recruited at a department of neurosurgery, in a university hospital in Kyoto, Japan. Their physician, who is a co-author, introduced them to the study. We included participants if they were aged 20 years and above, were lucid according to the Japan coma scale (The Joint Committee on Guidelines for the Management of Stroke, 2009), and were able to read and communicate in Japanese (we excluded those with symptoms of aphasia).

\section{Data Collection}

We collected data using semi-structured interviews and through the review of electronic medical records; we conducted interviews from May to December 2016 to explore participants' experiences of living with GBM, including their life stories. The interviews were focused mainly on perceptions of their symptoms, current disease status, and prognosis. 
Table 1. Participant Characteristics

\begin{tabular}{|c|c|c|c|c|}
\hline Characteristic & Ms. A & Ms. B & Mr. C & Ms. D \\
\hline Age & In her early thirties & In her late sixties & In his early thirties & In her early thirties \\
\hline Marital status & Unmarried & Unmarried & Unmarried & Engaged \\
\hline Living arrangement & $\begin{array}{l}\text { Living } \\
\text { with parents }\end{array}$ & Living alone & $\begin{array}{l}\text { Living } \\
\text { with parents }\end{array}$ & $\begin{array}{l}\text { Living } \\
\text { with parents \& fiancé }\end{array}$ \\
\hline $\begin{array}{l}\text { Work status } \\
\text { Diagnosis }\end{array}$ & $\begin{array}{l}\text { Employed } \\
\text { recurrent GBM }\end{array}$ & $\begin{array}{l}\text { Retired } \\
\text { recurrent GBM }\end{array}$ & $\begin{array}{l}\text { Unemployed } \\
\text { recurrent GBM }\end{array}$ & $\begin{array}{l}\text { On sick leave } \\
\text { recurrent secondary } \\
\text { GBM }\end{array}$ \\
\hline Tumor location & left frontal lobe & right angular gyrus & $\begin{array}{l}\text { right supplementary } \\
\text { motor area }\end{array}$ & right frontal lobe \\
\hline $\begin{array}{l}\text { Time since diagnosis } \\
\text { (months) }\end{array}$ & 20 & 15 & 112 & 9 \\
\hline PFS* (months) & 6 & 18 & 73 & 6 \\
\hline $\begin{array}{l}\text { Off treatment duration } \\
\text { before recurrence } \\
\text { (months) }\end{array}$ & 0 & 0 & 20 & 0 \\
\hline $\begin{array}{l}\text { Treatment history } \dagger \\
\text { (before TMZ mono- } \\
\text { therapy) }\end{array}$ & $\begin{array}{l}\text { surgical resection and } \\
\text { interstitial CT with } \\
\text { carmustine wafers } \\
\text { CRT with TMZ }\end{array}$ & $\begin{array}{l}\text { surgical resection and } \\
\text { interstitial CT with } \\
\text { carmustine wafers } \\
\text { CRT with TMZ }\end{array}$ & $\begin{array}{l}\text { surgical resection } \\
\text { CRT with TMZ }\end{array}$ & $\begin{array}{l}\text { biopsy prior to RT } \\
\text { against diffuse astro- } \\
\text { cytoma } \\
\text { surgical resection of } \\
\text { secondary GBM }\end{array}$ \\
\hline $\begin{array}{l}\text { TMZ monotherapy } \\
\text { (courses) }\end{array}$ & 6 & 17 & 47 & 7 \\
\hline
\end{tabular}

Note. $\mathrm{CT}=$ chemotherapy; $\mathrm{CRT}=$ chemoradiotherapy; $\mathrm{TMZ}=$ temozolomide; $\mathrm{RT}=$ radiotherapy

*PFS (Progression-free survival): The length of time during and after the treatment of a disease, such as cancer, that patient lives with the disease, but it does not worsen (National Cancer Institute, n.d.).

$\uparrow$ Treatment history: The standard treatment for newly diagnosed GBM is surgical resection to the extent feasible, followed by concurrent chemoradiotherapy with TMZ, followed by TMZ monotherapy (Stupp et al., 2005). Furthermore, some new approaches such as carmustine wafers and bevacizumab have been covered by insurance in Japan since 2013. TMZ monotherapy is required to be a maximum of six courses in Europe and the United States (Stupp et al., 2005), which probably reflects the medical situation; but in Japan, more than six courses may be covered by the health insurance system depending on adverse events (Hashimoto, 2016) and patients' choice.

tbiopsy before RT against diffuse astrocytoma: Ms. D was at the onset of secondary GBM after she received these treatments about seven years ago.

All participants were interviewed individually after using the Distress and Impact Thermometer (Akizuki, Yamawaki, Akechi, Nakano, \& Uchitomi, 2005) as a brief screening tool for adjustment disorders or major depression in cancer patients. We conducted interviews for about one hour at a time. Furthermore, the investigator interviewed participants several times (whenever possible, deferring to the participant's condition) when, during data analysis, the need arose for additional questions or analysis suggested a result that might differ from what subjects had reported.

\section{Data Analysis}

A thematic narrative analysis (Riessman, 2008) was performed to describe the individual experiences of illness in detail. It was challenging to recruit subjects because GBM is rare and symptoms vary widely depending on the location of the tumor. Thus, we anticipated that the experience of each patient would be unique. A thematic narrative analysis was focused on the content of speech exclusively and works from a case-centered model that enables to the researcher to 'keep a story 'intact' by theorizing from the case rather than component themes (categories) across cases" (Riessman, 2008, p. 53).

We used the thematic-narrative analysis procedure to analyze the data (Braun \& Clarke, 2006; Riessman, 1993, 2008). The interviewer transcribed interviews verbatim. Three investigators read the transcripts. Next, we analyzed each case ordering episodes into a chronological, biographical account, coding and identifying sub-themes and themes. In this analysis, we specifically focused on the experience of the period of PFS, from the end of the initial treatment to the recurrence. We then linked the themes among the four participant transcripts for a global analysis. We checked all themes presented in the data and individual analysis against each other and checked against the original data. Finally, we identified common themes and an overall main theme.

\section{Reflexivity and Rigor}

To ensure objectivity, we analyzed the interview data independently and then discussed the data 
with three investigators: a principal investigator and two collaborative researchers, both of whom had experience in qualitative research including narrative studies. Finally, we carefully triangulated the analyzed data with an expert researcher in palliative care nursing and qualitative research methods. Furthermore, to ensure the internal validity of the data interpretation, the participants confirmed the results as much as possible (Sakurai \& Kobayashi, 2005).

\section{Results}

\section{Patient Characteristics}

The participants were originally five adult patients with recurrent GBM, two males and three females, who had varying levels of physical function but were all-lucid. Five patients consented to participate; the authors due to clinical deterioration, leaving four subjects to complete the study, withdrew one subject. The average age of the four participants was 41.5 years $(S D \pm 17.8)$; the mean PFS was 28.7 months $(S D \pm 32.0)$. Table 1 shows the participant characteristics.

\section{Patient Experience}

We analyzed the four chronological biological accounts and identified themes in the experience of PFS per participant. We found four sub-themes from Ms. A, three sub-themes from Ms. B, two subthemes from Mr. C, and seven sub-themes from Ms. D. These themes, including the raw data, were compared and linked in a global analysis. As a result, three common themes emerged:

- Living vulnerable daily lives with uncertainty about the future and physical symptoms.

- Gaining a sense of normality and attempting to maintain it.

- Coping with bouts of pain while maintaining that sense of normality in daily life.

From these three themes, we distilled an overall main theme of the lived experience of GBM patients who had experienced PFS: Even in vulnerable and constrained daily lives, the aim was gaining a sense of stability - and maintaining it steadily — as far as possible, on their own. In the following sections, the details of those three common themes are described by referring to the raw data-translations from the participants' own words.

\section{Living vulnerable daily lives with uncertain- ty about the future and physical symptoms}

Participants knew the characteristics of GBM, including the fact of inevitable recurrence and prognosis. Furthermore, that knowledge made them aware of their limited life expectancy and uncertain future:
- $\quad$ "I heard from my sister about the life expectancy being two or three years. If it recurs, I do not know whether or not adequate treatment is available, but I did hear that it will be sure to relapse, so I will cross that bridge when I come to it" - Ms. B.

Moreover, participants had uncertainty about physical symptoms. They were always aware of the risks of sudden physical deterioration, such as a seizure caused by GBM, and they maintained epilepsy precautions. Furthermore, the uncertainty led to mental pain:

- 'I am not sure what will happen, I'm scared a bit..."-Ms. A.

- "I heard [that I will] never know when I collapse"-Ms. B.

- "[Regarding preventive behavior] I think I should do it because I cannot do many things if I collapse. It's important after all"-Ms. D.

Under such circumstances, when the participants thought about their uncertain future, recurrence, and prognosis, they tried to be positive and focused on the immediate situation:

- "I try to live every day with a smile as much as possible because my friends advised me that depression can accelerate the disease progression" -Ms. B.

- "I need positive thinking to get me through [this] illness. I try to think positively rather than negatively because I become more and more negative when I think negatively"Ms. D.

Each participant spent a stable everyday life in PFS although they realized that their disease would persist. Ms. A's daily life was almost the same as her premorbid life, without subjective symptoms. Ms. B was proud that she had overcome another life crisis, precipitated by acute hydrocephalus, before GBM. She reported a life wherein she perceived GBM as a serious disease but also valued her own current good physical condition. Mr. C spent a life without subjective symptoms after reoperation. Finally, Ms. D believed she was gradually getting better and had a plan to return to work. Overall, during the PFS stage, these participants hoped that their condition would stabilize, and they were able to think a little optimistically:

- "[I think,] just vaguely, well, I guess I might die, but... I was hospitalized because I was about to die. Hmm..., I overcame that, so I wonder if it will work out in the future" Ms. B.

- "After the second surgery, actually, my symptoms were getting better. I was okay after that, at that time"-Mr. C.

- "I thought it would last-a good condition like this" - Ms. D. 
In this way, they mentally distanced themselves from the dire prognosis that they had heard during hospitalization.

The participants experienced recurrence. All of them recognized that they would relapse someday, but Mr. C and Ms. D, who had exacerbated symptoms, felt the effect of relapse more acutely. Ms. A and Ms. B did not notice symptoms, as they stated the following in a bland tone:

- $\quad$ "I do not know [my condition] because I had no symptoms, but I realized it when I was shown my MRI image"-Ms. A.

- "Although I relapsed..."-Ms. B.

Mr. C and Ms. D both expressed their surprise at relapse. Although $\mathrm{Mr}$. C (the longest survivor) knew recurrence was frequently unavoidable, he was surprised by not only the recurrence but also motor dysfunction because he had spent about six years without any dysfunction or relapse. Meanwhile, Ms. D (shortest survivor) thought there was still a considerable amount of time before relapse:

- "Finally! It actually happened; I felt the time has come, but I never thought I would need a stick when I walk"-Mr. C.

- "I began to collapse, but I never expected the recurrence; I thought that relapse was still a little way off"-Ms. D.

These participants believed that they were getting better and regained their positive attitudes toward PFS. However, no one knew when the brain tumor would recur; its stability was always at risk, so they felt vulnerable in their daily lives. Even if the patients' condition is, good and regardless of whether the disease duration is long or short, their vulnerability remains as long as they have GBM. However, despite the vulnerability, they focused on the stable factors in their immediate situation rather than focusing on the thoughts of an uncertain future.

\section{Gaining a sense of normality and attempting to maintain it}

During PFS, participants did not have physical pain from symptoms, but they did experience side effects such as nausea, anorexia, and constipation within five days of chemotherapy (temozolomide monotherapy for five days every four weeks). Participants took constant precautions against epileptic seizures:

- "Actually, I had nausea during taking temozolomide. Because I never knew when I vomited, I was always in the kitchen whenever I vomited" - Mr. C.

In their PFS situation, they adapted their daily lives (e.g., meals, means of transportation) to accommodate their post-diagnosis situation. As the participants met the needs that changed from their premorbid life, they reported little trouble; participants complimented those around them for assisting.
Thereby, the participants obtained the subjective feeling that they were spending normal lives.

Ms. A did not experience any physical distress after being discharged from surgery. She returned to work on the fifth day after discharge. She has become able to work as well as she did before the occurrence of disease, receiving assistance from others in her surroundings:

- "I returned to work from the beginning of the week after discharge; I was fine. I cannot drive a car now, so my parents ride to work. I told my boss about my disease"-Ms. A.

Although Ms. B experienced a life crisis at the onset of GBM, the symptoms disappeared when she was discharged. Therefore, she could resume retirement life as she had lived before the occurrence of disease by substituting other means such as using personal deliveries for daily shopping and a microwave without gas:

- "I got well very much by rehabilitation after surgery, so I became rather normal. As rehab allowed my legs to move relatively quickly, and I could stand on one foot, actually I had not been having so much in trouble with daily life"-Ms. B.

Although Mr. C had intermittent numbness in left his leg, he could do carpentry as he did before diagnosis. That work convinced him he was no different from a healthy person:

- "The lumber was big, but I was strong enough for it. I did not feel that my physical strength had declined." - Mr. C.

Thus, three of the participants were able to realize normal functioning relatively early in PFS by keeping the ADL almost the same as before diagnosis. Ms. D thought she was no longer normal because surgery had lessened the fine motor function in her left hand. However, she felt she should be normal, and she pursued that goal by setting normal standards on her own. Because she went through a smooth recovery process supported by her loved ones, she was able to feel that she was normal:

- "Although I think perhaps healthy persons who are not sick also have a day like they feel down, feel a bit badly today, and feel like spoiling myself. I think the normal is maybe that I can manage those kinds of things well by a change of pace, increasing motivation. So, I was like I evaluated myself by just wondering how much the days like that would increase"-Ms. D.

\section{Coping with bouts of pain while maintaining that sense of normality in daily life}

All of the participants lived in PFS with independent ADL, without symptoms that reminded them of their GBM. Thus, during PFS, the participants felt themselves to be normal despite their GBM 
diagnosis. Still, at times, the participants suffered intractable pain. In particular, by leading a daily life that felt normal, they could feel they met even higher needs such as a self-actualization. The participants were aware of social pain. Saunders (1989) described the concept of pain by using the term "total pain," the sum of the physical, psychological, social, spiritual, and emotional elements that construct the total pain experience. Social pain refers to suffering to function every day, such as balancing the stressful situations of living with cancer, economic problems, work and resocialization, relationships with family, and care burdens (Japan Nursing Association, 2014). Participants suffered from increased living expenses including medical expenses, a sense of guilt for burdening their families, and limitations on their hobbies and entertainment.

Ms. A had no choice but to rely on her parents for driving a car; she was sorry for her parents. She felt disgusted about her narrow range of activities. Furthermore, she was also concerned about persistent hair loss from radiotherapy, and her body image was changing - a major problem especially for a woman in her $30 \mathrm{~s}$ :

- 'I'd like to drive extremely, as I can't ... No, not pain; actually, hair the loss was terrible - the most terrible thing. Around here, if you look closely, there is still no hair" -Ms. A.

Ms. B looked back at her life and mused about the reason for getting GBM:

- "I sometimes wonder if getting GBM is the punishment for many years of having [an] affair" -Ms. B.

At the same time, she said it brought up the most important memories of her life. Furthermore, when considering her death vaguely, she expressed spiritual pain. She had hoped to live longer, and she had a hard time motivating herself to sort through her belongings for her sisters.

- "I sometimes wonder if I deserved it. Well, actually, I just wonder if it cannot be helped, and I cannot be cured because I am old enough and living alone. But I just think that I have to settle for things until death"-Ms. B.

Mr. C reported no change regarding how to live, including his way of working. However, he did report economic distress from continuing the expensive chemotherapy. He was suffering from unstable symptoms that appeared when he got tired. Although sometimes he had regrettable experiences while working, economic pressure forced him to work. He was a young, independent ADL, and ostensibly able to work normally. He was suffering from social pain because it was difficult for him to find good employers and to access social welfare systems other than high-cost medical expense benefit. Mr. C reported why he had no choice but to quit chemotherapy in the fifth year:

- "I was no longer able to keep taking the medicine due to economic difficulty...but, I guess I have not been able to continue medicine [temozolomide] without receiving welfare like now. I am thinking that it cannot be helped though. Well, actually, my economic situation was so bad" - Mr. C.

Ms. D felt that she was recovering smoothly and was able to think positively. However, as for her dysfunctional left hand, she wanted to return to normal-her health status before her diagnosis. She had concerns:

- "Even though I know in my head that it [a complete cure] is impossible, I have gotten better. So, I cannot stop thinking, I wish I could return to my old self as premorbid." -Ms. D.

Furthermore, she was sorry to put a burden on her parents, and she worried about the future with her fiancé:

- 'I live in my parents' house. Well, I cannot afford an apartment fee; I feel like I cannot survive unless I live with my parents. I am sorry for my parents, though"-Ms. D.

\section{Discussion}

This study has cast a spotlight on how GBM patients perceive their life and how they cope with their illness. It was important for them to acquire and maintain a sense of normality on their own for adaptation in PFS. The results of this study present critical insights for nursing, as nurses seek to give patients quality time in PFS, by supporting their sense of normality with an interdisciplinary approach and lateral integration of care. We identified patients' narratives that may assist nurses to support patients, families, and clinicians for decision-making to prepare for the death to come.

\section{The Meaning of Their Uncertainty}

Patients perceived that the uncertainty due to an illness restricted their daily lives. Their uncertainty was caused by an unclear future, unpredictable medical condition and treatment effect, and unfamiliar illness, GBM itself. Our results support Mishel's explanation, "Uncertainty is generated by events characterized as vague, ambiguous, unpredictable, unfamiliar, in-consistent, or lacking information" (Mishel, 1984, p. 163). The prognosis of GBM forces patients to face the limitation of life left, which perpetuates uncertainty. However, participants were looking back on their life, considering what was now important for them in the light of continual uncertainty. Thus, the participants were aware of thinking posi- 
tively, focusing on their immediate lives, and setting their goals. Our results support Mishel's explanation that "the new view of uncertainty as a natural phenomenon is a new view of the world in which instability and fluctuation are natural and increase the person's range of possibilities" (Mishel, 1990, p. 261) Eventually, they may have approached adaptation to their changed lives, which are proposed as the end state achieved after coping with uncertainty (Mishel, 1990). The present results suggest that uncertainty, though fluctuating, is becoming weaker compared with what the participants felt at diagnosis. Although they kept relapse and death "in the back of their head," those images faded somewhat.

\section{The Meaning of the Sense of Normal: Equilibrium}

Participants in the present study had few or no physical symptoms except during chemotherapy. They experienced normalcy in their daily lives. The participants adjusted to their lives with GBM by delegating tasks to loved ones to make their everyday lives appear normal.

Johnson (1961) explained, "Equilibrium derives primarily from this stability-constancy concept. Equilibrium is viewed rather as a stabilized, but more or less transitory, resting state in which the individual is in harmony within himself and with his environment" (p. 65). Also, equilibrium takes place at points that the patient feels optimal gratification and minimal deprivation of his need-dispositions. We suggest that these patients felt the subjective sense of normalcy, that is, a sense of stability on their own through the re-establishment or maintenance of their equilibrium. However, it's likely that the equilibrium of GBM patients is fragile; their sense of stability included vulnerability.

\section{The Meaning of the Social Pain}

The participants often suffered social pain, although they had been performing ADLs independently, without GBM symptoms, while they gained a sense of stability during their PFS. Participants had social pain in common, such as the burden placed on families, the difficulty in returning to and continuing work, limitations on former hobbies and entertainment, concern about their future, and living expenses including medical expenses.

Notably, the three participants in their 30s (Ms. A, Mr. C, and Ms. D), had stronger social pain. Based on Erikson's (1980) psychosocial theory of development, they were at the stage between young and middle adult. During these stages, significant relationships with marital partners and friends and working are important. No matter how they had been obtaining and maintaining a sense of stability on their own, it was often hard for them to accomplish the developmental challenges of their stage due to GBM. Therefore, they were sometimes frustrated because they were strongly aware of the restrictions on their daily lives.

In comparison to the younger participants, Ms. B (in her late 60's) had more frequent remarks about spiritual pain, such as thinking about the meaning of illness and reflecting on her life. According to Erikson's (1980) theory, Ms. B was at the development stage of late adulthood, which involves accepting that one's life is one's responsibility through much reflection. Her spiritual pain was particularly related to the Erikson's development challenge of this stage.

\section{Implication for Nursing}

Any sense of stability among patients is likely to collapse because of living with an unpredictable disease like GBM. There is a delicate balance involved in maintaining a stable state of equilibrium, which can easily fluctuate and result in a feeling of vulnerability. Moreover, in PFS, subjects were trying to maintain equilibrium by themselves, sometimes by giving up something, although they received support from their loved ones.

As a premise, nurses are required to understand that patients are not necessarily optimistic just because they feel a sense of stability; they may also feel vulnerable at the same time. Also, through continuous involvement with patients, skilled nurses will grasp how each sense of stability fluctuates, and the nurses will provide support-oriented solutions, such as concrete and adequate information, that supports hope as much as possible. If at some point the patient is unable to feel hope, it would be helpful if we could connect the patient with a non-profit volunteer organization. However, currently in Japan, although there are organizations for children with intractable diseases such as Make a Wish® Japan (http://www.mawj.org/en/), for adults, there is only the cancer salon aimed at peer-support for survivors. Social welfare support has little available if patients have no or mild disability. Even if the condition is stable and the patient can perform ADL independently, hope may dwindle; thus, it is urgent that we develop support systems in light of the disease's severe prognosis. Otherwise, regardless of what the patient chooses, it is necessary to support their choice and encourage their confidence in it. These interventions will lead to developing a good relationship and symptom management and help patients prepare for the day to come, maximize inner stability, and optimize the choices they still have. Therefore, it is important for nurses to communicate effectively and integrate care laterally, including the hospital, outpatient settings, hospice, and rehabilitation settings. In fact, the Japanese government is promoting "community- 
based integrated care systems," to facilitate collaboration among various occupations and organizations. These systems are planned for completion by 2025 when Japan's aging population is estimated to reach about 30\% (Naruki, 2016). The system is still developing, and for these GBM patients in PFS, it is critical to promote the lateral integration of care as soon as possible.

\section{Limitations}

In this study, we interviewed patients after the recurrence of GBM. Although there is a possibility that their memories of what they narrated are not accurate, narrative inquiry is focused on the life history of patients to understand their subjective experience. Therefore, personal narrative is interpreted by the narrator at that time, is characterized by values and motivation, and is a subjective reality. As long as the narrative inquiry aims to understand subjective reality, internal validity is required rather than exploring the only true story (Sakurai \& Kobayashi, 2005). As we received suggestions during the analysis, we conducted as many additional interviews as possible to confirm the internal validity, depending on the condition of the patients. It was difficult to conduct additional interviews because of the physical and mental deterioration of the patients in several cases. GBM is a progressive disease, and the speed of progression after recurrence is often much faster than before its recurrence. Therefore, it cannot be said that we were able to collect all narratives. Furthermore, there were varying locations and symptoms of the tumor, duration of disease, and other factors in our participants. The tumor size and tumor location are significant because these affect the prognosis and various clinical symptoms. Because the tumor location in our participants allowed maximum safe surgical resection, it may have been less disabling. Meanwhile, there are many patients with some disability in PFS. Further analysis is needed to consider the differences in tumor location and symptoms, disease duration, genotype, and other factors.

\section{Conclusions}

Overall, the theme of the lived experience of illness in GBM patients focused on PFS can be expressed, even in vulnerable and constrained daily lives, the aim was gaining a sense of stability - and maintaining it steadily - as far as possible, on their own. Patients with GBM perceived restriction and vulnerability in daily life owing to chronic uncertainty. Because of responding to this uncertainty, participants were able to adapt to how their lives had changed from pre-illness and to attain a sense of stability on their own. They then attempted to maintain a certain, stable feeling of living in the present moment.
Nurses are required to understand and respect patients' situations. We suggest that in PFS, it is important for nurses to continue to engage with outpatients, develop a good relationship through conversations and symptom management, and help patients to prepare themselves for the end-of-life phase so that they can live as they wish and maintain an inner sense of stability. Thus, it is important for nurses to use effective communication in an interdisciplinary approach and lateral integration of care to meet the needs of GBM patients in PFS. However, further research is needed, which includes larger ongoing cohorts, to explore specific nursing interventions.

\section{Acknowledgments}

The authors would like to thank Rosemary Camilleri, Ph.D. for carefully proofreading the manuscript.

\section{Conflict of interest}

The author(s) declared no potential conflicts of interest concerning the research, authorship, or publication of this article.

\section{References}

Akizuki, N., Yamawaki, S., Akechi, T., Nakano, T., \& Uchitomi, Y. (2005). Development of an impact thermometer for use in combination with the distress thermometer as a brief screening tool for adjustment disorders and/or major depression in cancer patients. Journal of Pain and Symptom Management, 29, 91-99. https://doi.org/10.1016/j. ipainsymman.2004.04.016

Braun, V., \& Clarke, V. (2006). Using thematic analysis in psychology. Qualitative Research in Psychology, 3, 77-101. https://doi.org/10.1191/1478088706 qp063oa

Cahill, J. E., \& Armstorong T. S. (2011). Caring for an adult with a malignant primary brain tumor. Nursing, 41(6), 28-33. https://doi.org/10.1097/01. nurse.0000397930.50420.c6

Committee of Brain Tumor Registry of Japan. (2014). Report of the brain tumor registry of Japan (20012004) 13th edition. Neurologia MedicoChirurgica, 54(Suppl 1), 1-102. https://doi.org/ 10.2176/nmc.sup.2014-0001

Committee of Brain Tumor Registry of Japan. (2017). Report of the brain tumor registry of Japan (20052008) 14th edition. Neurologia MedicoChirurgica, 57(Suppl 1), 9-102. http://doi.org/ 10.2176/nmc.sup.2017-0001

Davies, N., Crowe, M., \& Whitehead, L. (2016). Establishing routines to cope with the loneliness associated with widowhood: A narrative analysis. Journal of Psychiatric and Mental Health Nursing, 23, 532-539. https://doi.org/10.1111/jpm. $\underline{12339}$ 
Erikson, E. H. (1980). Identity and the life cycle. New York: W. W. Norton.

Hashimoto, N. (2016). Standard therapeutic approach against gliomas with an implication for cancer immunotherapy. Journal of Kyoto Prefectural University of Medicine, 125, 173-183. Retrieved from http://www.f.kpum.ac.jp/k/jkpum/pdf/125/ 1253/hashimoto12503.pdf

Higuchi, J., Tanaka, K., Noguchi, M., \& Kusumi N. (2006). Relationship between higher brain dysfunction and ADL in brain tumor patients. Brain Nursing, 22, 307-313.

Iwata, T. (1998). What a doctor knew for the first time since becoming a terminal cancer patient; the fierce days a neurosurgeon fought brain tumor. Tokyo: Kadokawa Publishing.

Japan Nursing Association. (2014). Ministry of Health, Labor and Welfare Nursing training project involved in cancer care Palliative care education text for nurses (revised edition). Retrieved from https://www.nurse.or.jp/nursing/education/ganiry $\mathrm{o} / \# \mathrm{p} 2$

Johnson, D. E. (1961). The significance of nursing care. The American Journal of Nursing, 61(11), 63-66. https://doi.org/10.2307/3418646

Kamma, Y., Sato, R., \& Kuwabara, M. (2008). Suffering in primary malignant brain tumor patients with neurological dysfunction. Journal of Japanese Society of Cancer Nursing, 22, 77-85.

Kleinman, A. (1988). The illness narratives: Suffering, healing \& the human condition. New York: Basic Books.

Mishel, M. H. (1984). Perceived uncertainty and stress in illness. Research in Nursing \& Health, 7, 163171. https://doi.org/10.1002/nur.4770070304

Mishel, M. H. (1990). Reconceptualization of the uncertainty in illness theory. IMAGE: The Journal of Nursing Scholarship, 22, 256-262. https:// doi.org /10.1111/j.15475069.1990.tb00225.x

Narita, Y., \& Shibui, S. (2015). From data collection to clinical trials: Establish evidences of brain tumor. Japanese Journal of Neurosurgery, 24, 699-704.

Naruki, H. (2016). Examining the importance of and identifying methods to promote "integration" for structuring community-based integrated care systems. Journal of the National Institute of Public Health, $65,47-55$.

National Cancer Institute (n.d.). Definition of progressionfree survival - NCI Dictionary of Cancer Terms. Retrieved from https://www.cancer.gov/ publica$\underline{\text { tions/dictionaries/cancer-terms?cdrid }=44782}$
Philip, J., Collins, A., Brand, C. A., Moore, G., Lethborg, C., Sundararajan, V., ... Gold, M. (2014). "I'm just waiting...": An exploration of the experience of living and dying with primary malignant glioma. Supportive Care in Cancer, 22, 389-397. https:// doi.org/10.1007/ s00520-013-1986-1

Pompili, A., Telera, S., Villani, V., \& Pace, A. (2014). Home palliative care and end of life issues in glioblastoma multiforme: Results and comments from a homogeneous cohort of patients. Neurosurgical Focus, 37(6), E5. https://doi.org/10. 3171/2014.9.focus 14493

Riessman, C. K. (1993). Narrative analysis qualitative research methods series volume 30 . Newbury, CA: Sage. Publications, Inc.

Riessman, C. K. (2008). Narrative methods for the human sciences. Thousand Oaks, CA: Sage Publications, Inc.

Sakurai, A., \& Kobayashi, T. (2005). Life story interview qualitative research introduction. Tokyo: Serica Syobo.

Saunders, C., \& Baines, M. (1989). Living with dying: The management of terminal disease (2nd ed.). Oxford: Oxford University Press.

Sterckx, W., Coolbrandt, A., de Casterle, B. D., Van den Heede, K., Decruyenaere, M., Borgenon, S., ... Clement, P. (2013). The impact of a high-grade glioma on everyday life: A systematic review from the patient's and caregiver's perspective. European Journal of Oncology Nursing, 17, 107117. https://doi.org/10.1016/j.ejon.2012.04.006

Stupp, R., Mason, W. P., van den Bent, M. J., Weller, M., Fisher, B., Taphoorn, M. J., ... National Cancer Institute of Canada Clinical Trials Group. (2005). Radiotherapy plus concomitant and adjuvant temozolomide for glioblastoma. New England Journal of Medicine, 352, 987-996. https://doi. org/10.1056/NEJMoa043330

The Joint Committee on Guidelines for the Management of Stroke. (2009). Japanese guidelines for the management of stroke 2009. Retrieved from http://www. jsts.gr.jp/guideline/341.pdf

Tsumura, A., Yamazaki, A., \& Kamibeppu, K. (2010). Nursing strategies to help malignant glioma patients and their families make end-of-life decisions. Journal of Japan Academy of Nursing Science, 30(4), 27-35. https://doi.org/10.5630/jans. 30.4_27

Umeda, N., \& Iwata, H. (2015). Yuragi as experienced by middle-aged patients during Initial treatment of malignant glioma. Journal of Japanese Society of Cancer Nursing, 29(3), 29-39. 